MATEC Web of Conferences 47, 03019 (2016)

DOI: $10.1051 /$ matecconf/20164703019

(C) Owned by the authors, published by EDP Sciences, 2016

\title{
A Coupling Simulation Between Soil Scour and Seepage Flow by Using a Stabilized ISPH Method
}

\author{
Tomotaka Nogami ${ }^{1, a}$, Mitsuteru Asai $^{1}$, Kiyonobu Kasama ${ }^{1}$ and Taro Arikawa ${ }^{2}$ \\ ${ }^{1}$ Department of Civil Engineering, Graduate School of Engineering, Kyushu University, Nishi-ku, Fukuoka 819-0395, \\ Japan \\ ${ }^{2}$ Department of Science and Engineering, Chuo University, Bunkyo-ku, Tokyo 112-8551, Japan
}

\begin{abstract}
In 2011, the example that breakwaters collapsed because of the basic ground's destabilization was reported by Tohoku-Kanto earthquake tsunami. Fluid-Structure-Soil coupling simulation is desired for a systematic comprehension of the breakwater collapse mechanism, and it may help to develop next disaster prevention method. In this study, A particle simulation tool based on the SPH has been modified and improved to analyze seepage flow and soil scouring. In seepage flow analysis, as a first step, this simulation treat the surface flow and seepage flow interactions by using governing equation. In the scouring analysis, soil scour is judged by an empirical criteria based on quicksand quantity formula.
\end{abstract}

\section{Introduction}

In 2011, Tohoku-Kanto earthquake, tsunami caused serious damage to the port structures such as a breakwater and seawalls. Damage mechanisms of these structures have been studied in the past, and there are mainly three causes; I. Horizontal force due to the water level difference between the front and rear breakwater, II. Soil scour and erosion behind the seawall during overflow and III. Piping destruction associated with the decline of the bearing capacity by seepage flow. Fluid-Structure-Soil coupling simulation is desired for a systematic comprehension of the seawall collapse mechanism, and it may help to develop next disaster prevention method. In this study, a particle simulation tool based on the SPH has been developed to solve seepage failure problem and scouring phenomenon. In this study, as a first step, surface flow and seepage flow are described by the same governing equations, and an SPH formulation has been developed. After that, efficiency and adequacy of the proposed simulation technique has been validated through an application to one test. On the other hand, by using the empirical judging equation, we implemented the numerical analysis to replicate phenomenon of scouring in the back of breakwater.

\section{Seepage Flow Analysis}

In this part, as a first step, surface flow and seepage flow are described by the same governing equations, and an SPH formulation has been developed.

\footnotetext{
${ }^{a}$ Corresponding author : t_nogami@doc.kyushu-u.ac.jp
} 


\section{MATEC Web of Conferences}

\subsection{Governing equation}

Fluid flowing outside the soil is generally modeled as Newtonian fluid, and described Navier-Stokes equation as:

$$
\frac{D v}{D t}=\frac{1}{\rho^{w}}\left(-\nabla P+\rho^{w} g\right)+\left(v_{w}+v_{T}\right) \nabla^{2} v
$$

In addition to the above equation, the mass conservation law was also used as governing equation.

$$
\frac{D \rho}{D t}+\rho \nabla \cdot \boldsymbol{v}=0
$$

Here, $\mathrm{v}, \mathrm{P}, \mathrm{g}$ is water's velocity, pressure, gravitational acceleration. $\mathrm{vw}$ is the kinematic viscosity coefficient of water and $\rho w$ is specific density. $v T$ is the kinematic eddy viscosity coefficient of water. On the other hand, the soil is generally described Darcy's law as:

$$
\frac{\mu}{K} \boldsymbol{v}=-\nabla P+\rho^{w} \boldsymbol{g}
$$

Here, $\mathrm{K}$ is a specific permeability coefficient in soil mound and $\mu$ is viscosity. In a field of the hydrology, the Darcy's law has been used as an equation of motion widely. However, it is reported that Darcy's law is not established in a flow field with a low Reynolds number, and then the nonDarcy flow rule is adapted as a model of seepage flow in this study. The traditional Extended Darcy's law suggested by Forchheimer is given by:

$$
\frac{\mu}{K} \boldsymbol{v}+\frac{\rho^{w} C}{\sqrt{K}} \boldsymbol{v}|\boldsymbol{v}|=-\nabla P+\rho^{w} \boldsymbol{g}
$$

The above equation is not enough to simulate collapse behavior of seawall and breakwater structure, and it is necessary to unify the governing equations both for the free surface flow and seepage flow. Akbari equation $[1,2]$ is introduced as an unified governing equation as follows:

$$
\frac{C_{r}(\varepsilon)}{\varepsilon} \frac{D \boldsymbol{v}_{D}}{D t}=-\frac{1}{\rho^{0}} \nabla P+v_{E}(\varepsilon) \nabla^{2} \boldsymbol{v}_{D}-a(\varepsilon) \boldsymbol{v}_{D}-b(\varepsilon) \boldsymbol{v}_{D}\left|\boldsymbol{v}_{D}\right|+\boldsymbol{g}
$$

Here, ${ }^{v} D$ is Darcian velocity or average velocity, and it has a relationship with the regular velocity $v$ as ${ }^{v} D^{=\varepsilon v}$. Meanwhile, $\varepsilon$ is porosity in the ground. Various coefficients in the Akbari's equation are given as a function of the porosity and their expressions of relations are as follows.

$$
\begin{gathered}
C_{r}(\varepsilon)=1+0.34 \frac{1-\varepsilon}{\varepsilon}: \text { inertia coefficient } \\
v_{\mathrm{E}}(\varepsilon)=\frac{v_{w}+v_{T}}{\varepsilon}: \text { effective viscosity coefficient } \\
a(\varepsilon)=\alpha_{c} \frac{v_{w}(1-\varepsilon)^{2}}{\varepsilon^{3} D_{50}^{2}}: \text { linear coefficient } \\
b(\varepsilon)=\beta_{c} \frac{(1-\varepsilon)}{\varepsilon^{3} D_{50}}: \text { nonlinear coefficient }
\end{gathered}
$$

$D_{50}$ is expressed the average particle size of ground materials and the value of $\alpha_{c}, \beta_{c}$ set $\alpha_{c}=1000$ and $\beta_{c}=1.1$. 


\subsection{ISPH method}

In this paper, smoothed particle hydrodynamics ( $\mathrm{SPH}$ ) method was adopted to solve the Akbari's unified equation for free surface and seepage flow. The basic concept in SPH method is that any function $\phi$ attached to particle " $i$ " at a position $\boldsymbol{r}_{\boldsymbol{i}}$ is written as a summation of contributions from neighbor particles. Note that, the triangle bracket $<\phi_{i}>$ means SPH approximation of a function $\phi$.

$$
\varphi\left(r_{i}\right) \approx\left\langle\varphi_{i}\right\rangle=\sum_{j} \frac{m_{j}}{\rho_{j}} \varphi_{j} W\left(r_{i j}, h\right)
$$

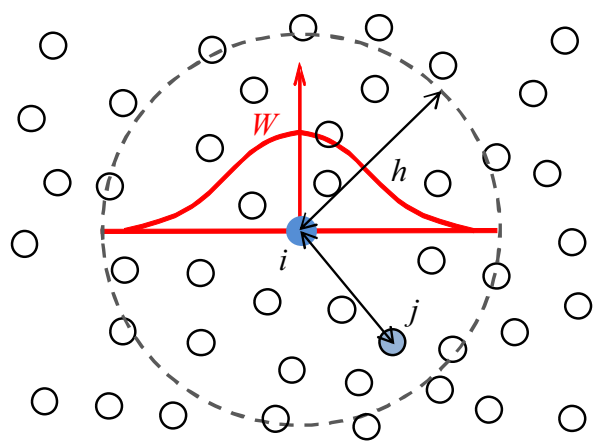

Figure 1. Particle placement and influence radius in the SPH method.

The Laplacian of the function can be assumed by using the above defined SPH approximation as follows:

$$
\left\langle\nabla^{2} \varphi_{i}\right\rangle=\sum_{j} m_{j}\left(\frac{\rho_{i}+\rho_{j}}{\rho_{i} \rho_{j}} \frac{\boldsymbol{r}_{i j} \cdot \nabla W\left(r_{i j}, h\right)}{\boldsymbol{r}_{i j}^{2}+\eta^{2}}\right)\left(\varphi_{i}-\phi_{j}\right)
$$

The main concept in an incompressible SPH method [3] for Navier-Stokes equation is to separate the governing equations of the incompressible fluid by using projection method. In this method, the pressure is calculated implicitly and the velocity fields are updated explicitly. In this study, the same idea of ISPH for the Navier-Stoke equation is applied to solve the unified equation as follows:

$$
\begin{gathered}
\frac{D \bar{\rho}}{D t}+\bar{\rho} \nabla \cdot \frac{\boldsymbol{v}_{D}}{\varepsilon}=0 \\
\frac{C_{r}(\varepsilon)}{\varepsilon} \frac{D \boldsymbol{v}_{D}}{D t}=-\frac{1}{\bar{\rho}} \nabla P+\frac{\boldsymbol{g}}{\varepsilon}+v_{E}(\varepsilon) \nabla^{2} \boldsymbol{v}_{D}-a(\varepsilon) \boldsymbol{v}_{D}-b(\varepsilon) \boldsymbol{v}_{D}\left|\boldsymbol{v}_{D}\right|
\end{gathered}
$$

Here, the density can be assumed as a constant value because of the incompressible assumption. Equation (12) can change as follows:

$$
\nabla \cdot v_{D}=0
$$

In the incompressible SPH method and treatment, the final pressure Poisson equation is given by:

$$
\left\langle\nabla^{2} P_{i}^{n+1}\right\rangle=\frac{C_{r}\left(\varepsilon_{i}\right)}{\varepsilon_{i}} \frac{\rho_{i}^{0}}{\Delta t}\left\langle\nabla \cdot v_{D i}^{*}\right\rangle
$$

During numerical simulation, the 'particle' density may change slightly from the initial value because the particle density is strongly dependent on particle locations in the SPH method. If the particle distribution can keep almost uniformity, the difference between 'physical' and 'particle' 


\section{MATEC Web of Conferences}

density may be vanishingly small. In other words, accurate SPH results in incompressible flow need to keep the uniform particle distribution. For this purpose, the different source term in the pressure Poisson equation can be derived using the 'particle' density. In stabilized ISPH method [4], the pressure Poisson Equation (15) reformulated as:

$$
\left\langle\nabla^{2} P_{i}^{n+1}\right\rangle \approx \frac{C_{r}\left(\varepsilon_{i}\right)}{\varepsilon_{i}}\left(\frac{\rho_{i}^{0}}{\Delta t}\left\langle\nabla \cdot \boldsymbol{v}_{D i}{ }^{*}\right\rangle+\alpha \varepsilon_{i} \frac{\rho_{i}{ }^{0}-\left\langle\rho_{i}^{n}\right\rangle}{\Delta t^{2}}\right)
$$

where $\alpha$ is relaxation coefficient, $\boldsymbol{u}_{i}{ }^{*}$ is temporal velocity and triangle bracket $<>$ means SPH approximation. Note that this relaxation coefficient is dependent on the time increment and the particle resolution. Then, the reasonable value can be estimated by the simple hydrostatic pressure test using the same settings on its time increment and the resolution. In this study, $\alpha=0.01$.

\subsection{Validation test}

In validation, the experimental test ralated to seepage problem in breakwater structure is simulated. This experiment was conducted by Kasama et al. [5]. The penetration collapse of the breakwater in case given water level difference was observed. The water level difference $\Delta \mathrm{h}$ is selected as $40 \mathrm{~mm}$, $80 \mathrm{~mm}, 120 \mathrm{~mm}, 145 \mathrm{~mm}$, and only in case of $145 \mathrm{~mm}$ the mound collapsed. Experimental outline and analysis model is shown in Figure 2 and Figure 3. In Figure 2, blue points are water pressure gauge and Figure 4 shows their number. In this analysis, the particle distance $d_{0}=1 \mathrm{~cm}$, time increment $\Delta t=$ $0.001 \mathrm{~s}$ and the total numbers of particles are about 8 hundred thousand. Piezo water head shown below was used for a comparison between experimental and analysis. In analysis, $\Delta \mathrm{h}$ is selected $145 \mathrm{~mm}$ as the biggest water level. The analysis result of comparison of piezo water head in case of $145 \mathrm{~mm}$ is shown in Figure 5. From this comparison, the value of the analysis is slightly different.

$$
\text { Piezo }=\frac{P}{\rho \boldsymbol{g}}+\Delta z
$$

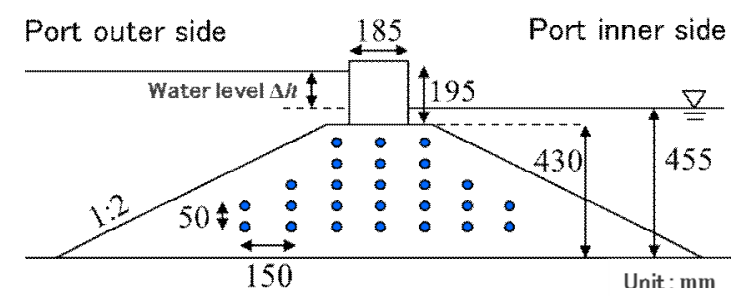

Figure 2. Experimental outline.

Figure 3. Analysis result.

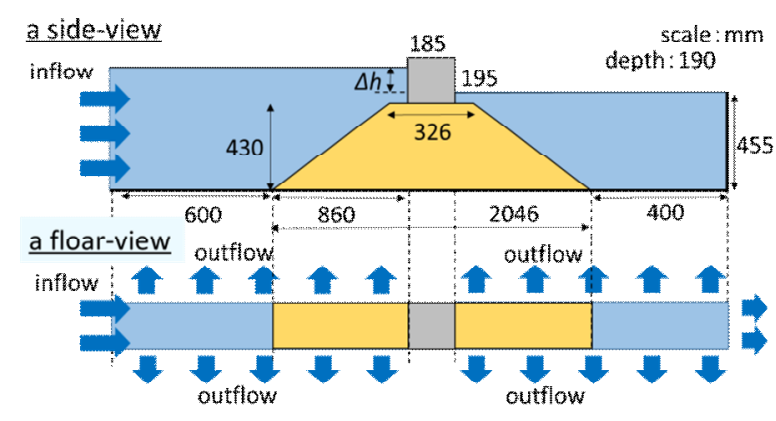




\section{IConCEES 2015}

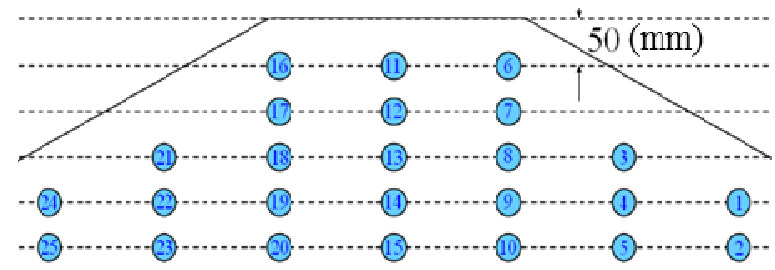

Figure 4. Water pressure gauge number.

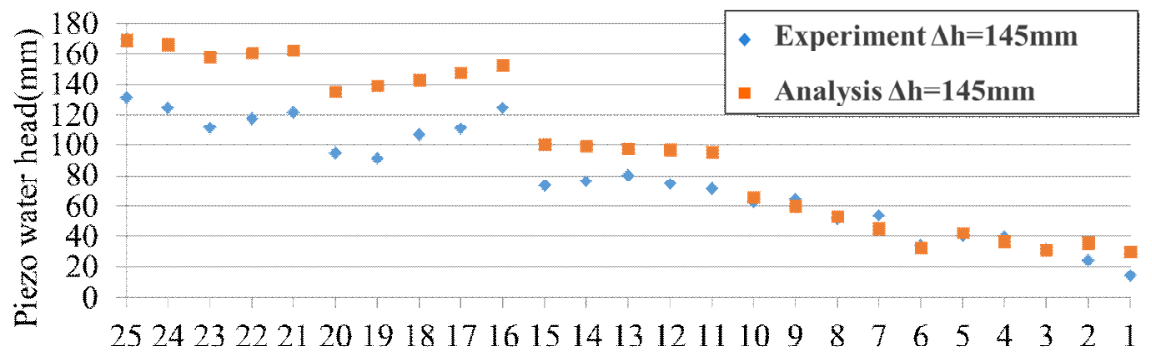

Number of water pressure gauge

Figure 5. Analysis result (Piezo water head).

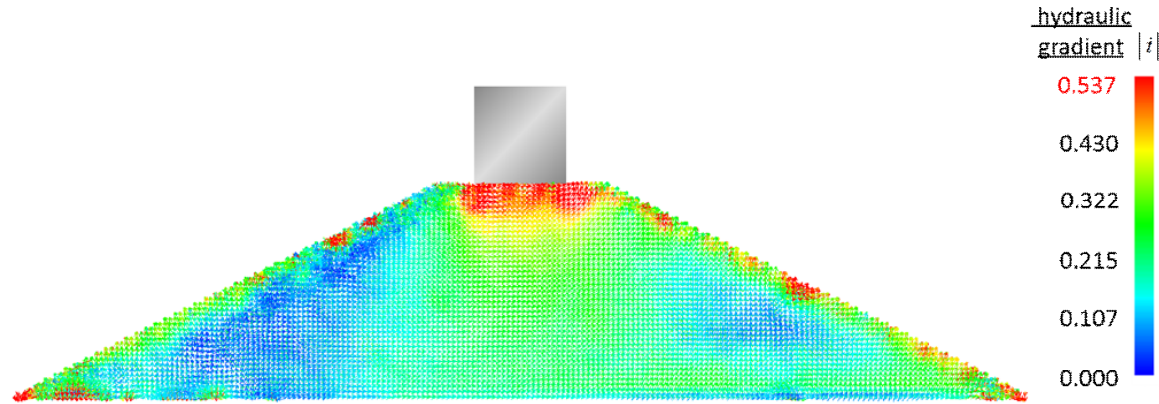

Figure 6. Analysis result (hydraulic gradient).

However, an analysis result behavior shows a good agreement with the hydraulic experiment test. Finally, the seepage failure can be estimated by using our simulated hydroulic gradient by refering to its limitation as shown in Figure 6. In the figure, red color region shows the possible domain for failure by seepage flow.

\section{Seepage Flow Analysis}

\subsection{Scouring judging formula}

Soil scour may depend on shear velocity of fluid flow and particle size on the see floor. In this study, soil scour is judged by an empirical criteria based on quicksand quantity formula [6]. The velocity of threshold of sediment movement is shown as follows. 


$$
\begin{gathered}
u_{\text {limit }}=\sqrt{\frac{2 A_{3}\left(\mu_{f} \cos \theta-\sin \theta\right)\left(\rho_{s p} / \rho_{l}-1\right) g d}{\left(C_{D}+\mu_{f} C_{L}\right) A_{2}}}: \text { water flow slope downward } \\
u_{\text {limit }}=\sqrt{\frac{2 A_{3}\left(\mu_{f} \cos \theta+\sin \theta\right)\left(\rho_{s p} / \rho_{l}-1\right) g d}{\left(C_{D}+\mu_{f} C_{L}\right) A_{2}}} \quad \text { water flow slope upward }
\end{gathered}
$$

Here, $A_{3}$ is three-dimensional shape coefficient of the soil particle, $\mu_{f}$ is coefficient of static friction, $\theta$ is slope inclination, $\rho_{s p}$ is density of soil particle, $C_{D}$ is drag coefficient, $C_{L}$ is lift coefficient, $A_{2}$ is two-dimensional shape coefficient of the soil particle. Once soil particle meets the above condition, the fixed soil particle is shited as a moving particle based on fluild flow with a density as the soil material.

\subsection{Validation test}

By using the empirical equation based on the correlation between shear velocity and sediment discharge, we implemented the numerical analysis to replicate phenomenon of scouring in the back of breakwater. As analysis model, the experiment that was conducted by Arikawa [7] is adapted. In this experiment, overflow of tsunami is reproduced by hydraulic test. Water level difference $20 \mathrm{~cm}$ is selected and validated in this study. Experimental outlines and Analysis models are shown in Figure 7 and Figure 8. The analysis result of comparison of each time and scouring shape in $15 \mathrm{~s}$ is shown in Figure 9 and Figure 10. From this comparison, our model can estimate tendency and the depth of the soil souring quantitatively, but it still has some difference with experimental result especially in the shape of soil souring. In this study, soil particle's sedimentation after soil scour occured doesn't be considered yet, then the shape after the soil scouring couldn't be reproduced. In the future work, this analysis would be expanded to be able to consider soil sedimentation.

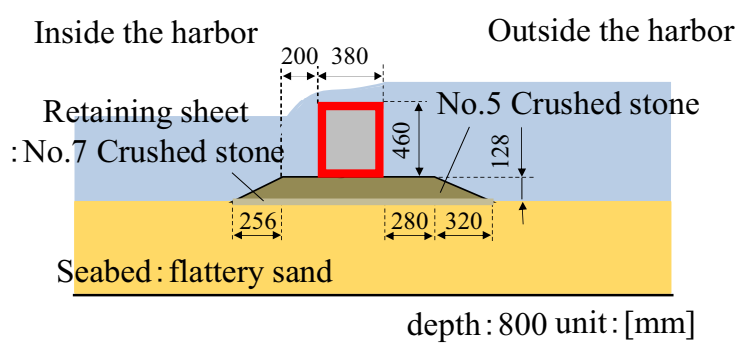

Figure 7. Experimental outline.

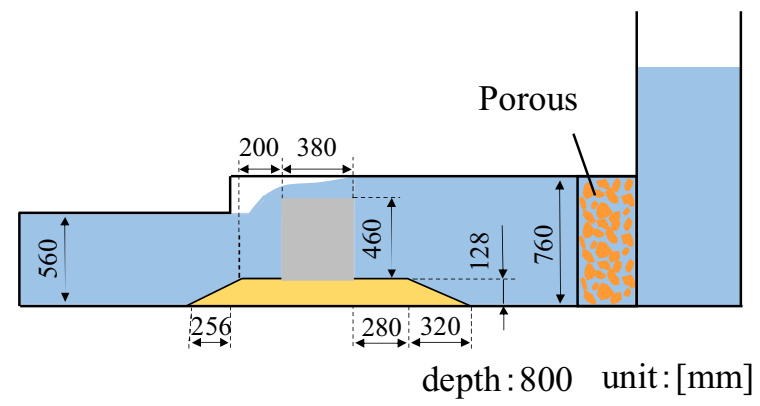

Figure 8. Analysis model. 
Figure 9. Analysis result.
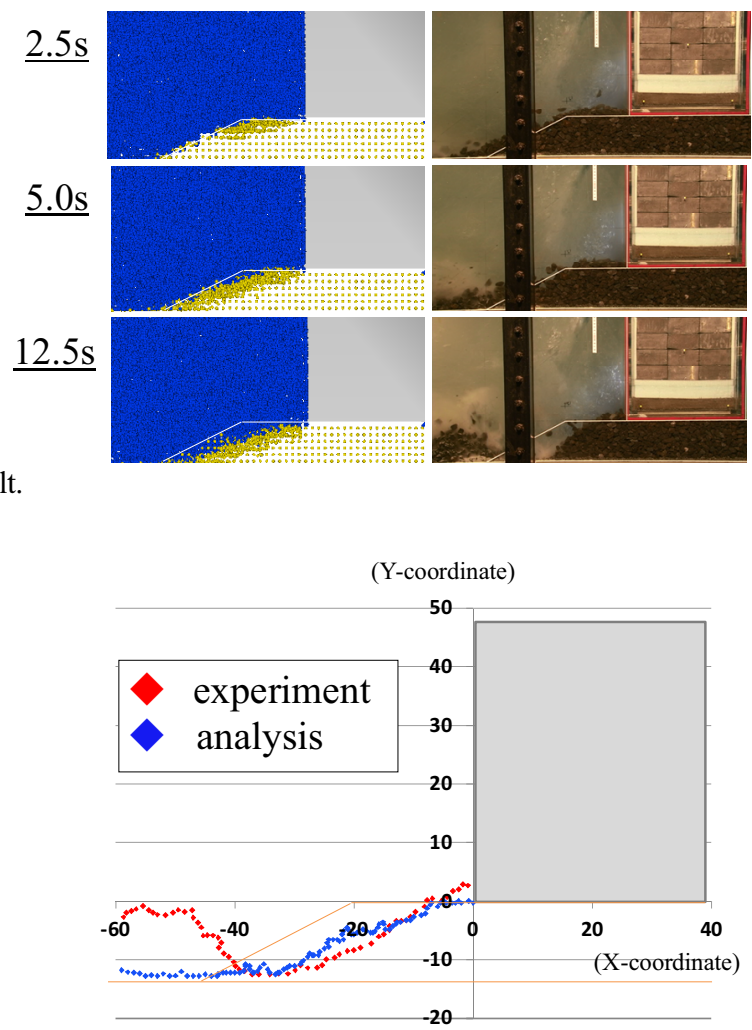

Figure 10. Scouring shape (15s).

\section{Summary}

In this study, a particle simulation tool based on the SPH has been developed to solve both of the seepage failure problem and the soil scour problem. In seepage flow analysis, a unified government equation which can model both of free surface flow and seepage flow is applied to a particle simulation. From the verification and validation test, the effect of porosity and reasonable surface flow and seepage flow behavior has been given. In scouring analysis, scouring phenomenon was reproduced by using the scouring judging formula. In the future works, this analysis method would be expanded to soil scour and seepage flow coupling simulator.

\section{Acknowledgement}

This work was supported by JSPS KAKENHI Grant Numbers 26282106, 15K12484.

\section{References}

[1] H. Akbari, Modified moving particle method for modeling wave interaction with multi layered porous structures, Coast. Eng., 89, 1-19, (2014).

[2] H. Akbari and M.M. Namin, Moving particle method for modeling wave interaction with porous structures, Coast. Eng., 74, 59-73, (2013).

[3] M.A.A.A. Abdelraheem, An improved incompressible smoothed particle hydrodynamics to simulate fluid-soil-structure interactions, Kyushu University, (2012). 


\section{MATEC Web of Conferences}

[4] M. Asai, A.M. Aly, Y. Sonoda and Y. Sakai, A stabilized incompressible SPH method by relaxing the density invariance condition, Int. J. for Applied Mathematics, 2012, (2012).

[5] K. Kasama, K. Zen and Y. Kasugai, Model experiment for the instability of caisson-type composite breakwater under tsunami condition, Proceedings of Coastal Engineering JSCE, (2013).

[6] H. Gotoh, Computational Mechanics of Sediment Transport, Morikita Shuppan Co. Ltd., (2004).

[7] T. Arikawa, S. Oikawa and S. Moriyasu, Stability of the Breakwater with Steel Pipe Piles under Tsunami Overflow, Independent Administrative Institution, Port and Airport Research Institute, Japan, (2015). 